\title{
A PSpice Circuit Model for Single-Photon Avalanche Diodes
}

\author{
Yuchong Tian, Junjie Tu, Yanli Zhao \\ Wuhan National Laboratory for Optoelectronics, Huazhong University of Science and Technology, Wuhan, China \\ Email: yanlizhao@mail.hust.edu.cn
}

How to cite this paper: Tian, Y.C., Tu, J.J. and Zhao, Y.L. (2017) A PSpice Circuit Model for Single-Photon Avalanche Diodes. Optics and Photonics Journal, 7, 1-6. https://doi.org/10.4236/opj.2017.78B001

Received: March 15, 2017

Accepted: August 7, 2017

Published: August 10, 2017

\begin{abstract}
In this paper, we present an improved circuit model for single-photon avalanche diodes without any convergence problems. The device simulation is based on Orcad PSpice and all the employed components are available in the standard library of the software. In particular, an intuitionistic and simple voltage-controlled current source is adopted to characterize the static behavior, which can better represent the voltage-current relationship than traditional model and reduce computational complexity of simulation. The derived can implement the self-sustaining, self-quenching and the recovery processes of the SPAD. And the simulation shows a reasonable result that the model can well emulate the avalanche process of SPAD.
\end{abstract}

\section{Keywords}

Single-Photon Avalanche Diodes, Circuit Model, Orcad PSpice

\section{Introduction}

Weak signal detection has been well developed over the past several decades [1]. There are many active devices applied to it, typically photo multiplier tubes (PMT), avalanche photodiodes (APD) etc. However it's unpractical to adapt PMT and ordinary APD in many areas. Because PMT needs complex circuit and high bias voltage to drive it, while the gain of ordinary APD is too low to reach the requirement of detecting single photon. But single-photon avalanche diodes(SPAD) can be operated in Geiger model (the reverse bias voltage beyond breakdown voltage) with high gain and sensitivity, Which make them widely utilized in many areas such as quantum cryptography [2], biological imaging [3], laser ranging [4], ultrasensitive spectrum measurement [5], and fluorescent decays [6], etc. Long-time operation in Geiger model will cause irreversible dam- 
ages to SPAD, for this reason, an external circuit is required to quench the avalanche process and reset the reserve biased voltage after each pulse. The common quenching and recharging circuit are passive quenching circuit (PQC), active quenching circuit (AQC) and mixed quenching circuit (MQC) [7]. Building model is a significant and effective way to study SPAD, which can improve device performance, shorten development cycle and lower costs.

A traditional way to build model is using simple circuit to express SPAD behaviors. A basic SPAD model is proposed in [8], which consists of a breakdown voltage source, a fixed diode resistance, a n-MOS switch and two fixed capacitances. The model is simple enough but can't guarantee accuracy. Based on the basic model, an improved Spice model was presented in [9]. All of the components are available in standard Orcad PSpice library, a piecewise linear voltage generator is adopted to replace the fixed source and resistance in traditional model. It certainly improves the simulation accuracy, but falls into convergence problems for the current-voltage (I - V) curve is not continuously differentiable. A model established in hardware describe language overcome the convergence problems [10], but it not intuitionistic and convenient for designer to use.

In this paper, an enhanced SPAD model is proposed with Orcad PSpice. A voltage controlled current source (VCCS) is used to replace the piecewise linear voltage generator in [9]. It can intuitively express the relationship between voltage and current, and reduce the computational complexity for adopting polynomial fitting to rebuild the static I - V curve. What's more important is the use of continuously differentiable curve will not cause convergence problems.

\section{Enhanced SPAD Circuit Model}

In this paper, we present an improved SPAD model based on the traditional models implemented in [8] [9]. As shown in Figure 1 is the structure of the model. It consists of a VCCS $I_{S P A D}$, replaced the piecewise linear voltage generator in [9], a junction capacitance $C_{A C}$ two stray capacitances $C_{C S}$ and $C_{A S}$ two voltage-controlled switches $S_{1}$ and $S_{\text {TRIG }}$ a current-controlled switch $S_{S E L F}$ two resistances $R_{1}$ and $R_{2}$, three external interfaces "Cathode", "Anode" and "Photon", and one internal port "Sub". All these component are from the standard library of Orcad PSpice and can be easily used by designers. The core parts of the SPAD model are $I_{S P A D}$ and capacitances, the former mimics the static behavior while the latter emulates the dynamic behavior. Among them, junction capacitance controls the quenching and recovery time, while stray capacitances affect the signal extraction. Moreover, the switch $S_{1}$ guarantees that the avalanche process can only be triggered in Geiger model, avoiding incident photon. With the $S_{T R I G}$ and $S_{S E L F}$ the model can implement avalanche processes.

As shown in Figure 2, the I-V measurement points is from a typical Si SPAD and the breakdown voltage $V_{B}$ is about $21.54 \mathrm{~V}$ [11]. The SPAD I-V characteristics in Geiger model is nonlinear. So, it is inaccurate to use a steady DC source to simulate it. Using a piecewise linear curve is a more accurate way to reproduce 


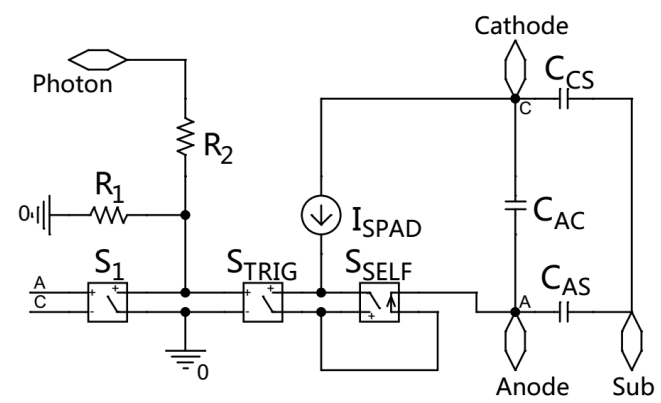

Figure 1. The improved SPAD model. The "Sub" connected to the ground.

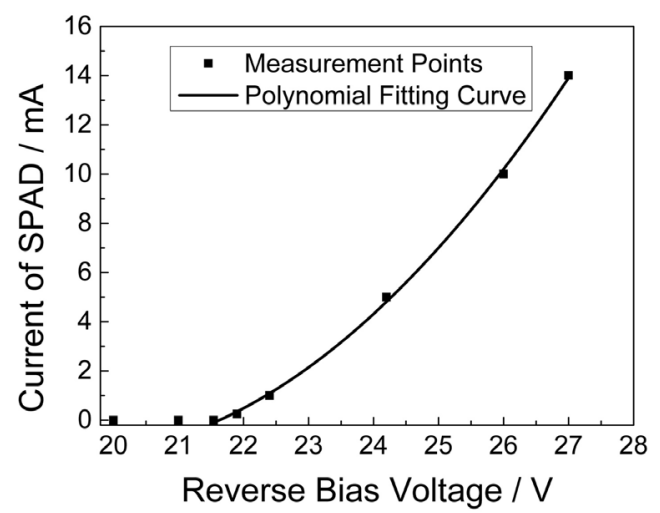

Figure 2. The measurement of I - V characteristic and the 2nd order Polynomial fitting curve.

the static I - V behavior, however, since the curve is not continuously differentiable function, the convergence problems will cause terrible consequences. Therefore, this paper presents a new means to rebuild the character, through making polynomial fitting of the measurement points and encoding the parameters into a VCCS to characterize the static I - V relationship. It has the following advantages: 1) The fitting curve is a continuous differentiable function that will avoid the convergence problems in simulation. 2) VCCS is an intuitionistic representation of I-V relationship than voltage generator. 3) The codes input to $I_{S P_{A D}}$ are very simple which have no complicated judgment statement as show in Equation (1)

$$
a_{0}+a_{1} * b_{1}+a_{2} * b_{2}^{2}+\cdots+a_{n} * b_{n}^{n}
$$

Among them, " $b_{n}$ " is nth order of the voltage $I_{\text {SPAD }}(\mathrm{n}=1,2,3 \ldots)$; " $a_{0}$ " is the constant term, and " $a$ " are the coefficients of corresponding terms $(n=1,2$, 3...). The coefficients are calculated in MATLAB, from which also reducing the computational complexity. The PSpice properties of $I_{S P_{A D}}$ is shown in Table 1. The PSpice Template is written in algebraic equation macro model and the property "Func" represents the fitting polynomial.

The model is operated with PQC, which supply voltage $V_{A}$ is beyond SPAD breakdown voltage $V_{B}$. Initially, the SPAD is reverse biased at $V_{A}$, the voltage-controlled switch $S_{\text {TRIG }}$ and the current-controlled switch $S_{S E L F}$ is open, and 
Table 1. The PSpice properties implemented in $I_{S P A D}$.

\begin{tabular}{cc}
\hline Property & Value \\
\hline PSpice Template & $\mathrm{G}^{\wedge} @$ REFDES $\%+\%-$ VALUE $=\{@$ Func $\}$ \\
Func & $@ \mathrm{a}_{0}+\mathrm{a}_{1}{ }^{*} @ \mathrm{~b}_{1}+\mathrm{a}_{2}{ }^{*} @ \mathrm{~b}_{2}$ \\
$b_{1}$ & $\mathrm{~V}(\%+, \%-)$ \\
$b_{2}$ & $\mathrm{~b}_{1}{ }^{*} \mathrm{~V}(\%+, \%-)$ \\
$a_{0}-a_{n}$ & Input by the designer \\
\hline
\end{tabular}

there is no current flow through the diode model. A short pulse source simulates single photon to trigger the "Photon" input port. Once triggered, the switch $S_{\text {TRIG }}$ gets close due to the voltage division of $R_{1}$ and $R_{2}$. Then, the current sharply rises to macroscopic level, which beyond the threshold level of $S_{S E L F}$ and makes it closed. With the suppression of QPC, the current will drop slowly but not be cut off unless it is lower than threshold, even $S_{\text {TRIG }}$ is opened. When the current is lower than the threshold, the $S_{S E L F}$ opens. With the charging of capacitances the model returns back to the initial state, waiting for the next photon trigger.

\section{Model Simulation and Validation}

In the simulation, this paper uses the I - V characteristics of a Si SPAD reported in [11] to construct fitting function. According to the characteristics of the measurement points, we use 2nd order polynomial to fit the static current-voltage curve as show in Figure 2. The current limiting resistance $R_{L}$ in PQC is 100 $\mathrm{k} \Omega$ and the signal extracting resistance $R_{S}$ is $50 \Omega$.

Figure 3(a) reports the avalanche signal voltage across $R_{S}$ as a function of the time when excess voltage $V_{e x}\left(V_{e x}=V_{A}-V_{B}\right)$ increases from $3 \mathrm{~V}$ to $5 \mathrm{~V}$. As shown in figure, the avalanche voltage increases as the Vex increases. The phenomenon of avalanche signal is as we expected, a burst rise is generated in the first place, then it drops gradually until the current is lower than threshold. It significantly simulates the self-sustaining and self-quenching process.

Figure 3(b) shows the cathode voltage at the photon pulse frequency of 200 $\mathrm{kHz}$, from which we can observe the recovery process after each avalanche triggering. Initially, the voltage is at a high level. Once triggered, the voltage rapidly falls down to a low level and gradually recovers to the high level during a few microseconds due to the recharge of capacitances. This period is the recovery time of every avalanche effect.

\section{Conclusion}

We proposed a SPAD model based on Orcad PSpice and all the components are available in the standard PSpice library. A voltage-controlled current source is adopted, which can overcome the convergence problems, intuitionistic represent the I-V relationship, and decrease the amount of code to reduce the computational complexity. Cooperated with the PQC, the model can certainly simulate 


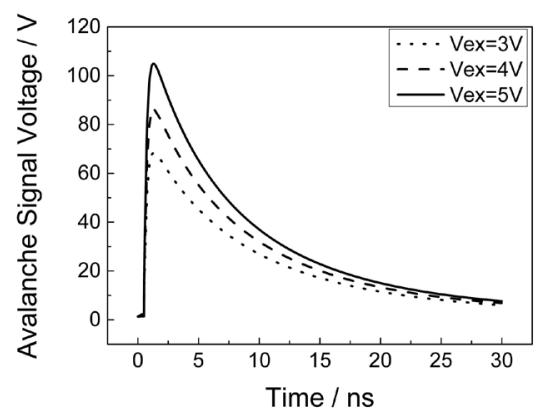

(a)

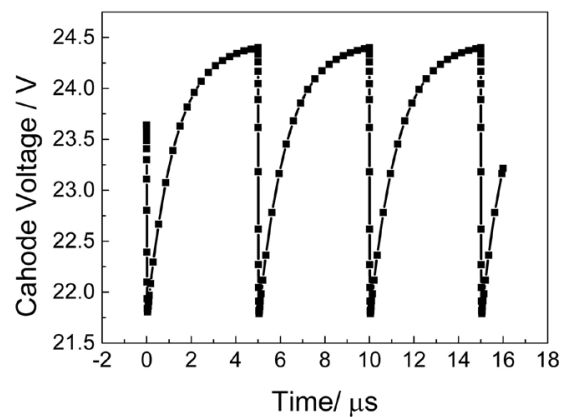

(b)

Figure 3. (a) The avalanche signal voltage through $R s$ at different excess voltage Vex; (b) The voltage waveform of cathode at the photon pulse frequency of $200 \mathrm{kHz}$.

static and dynamic behaviors, including self-sustaining, self-quenching and recovery process.

\section{References}

[1] Meng, X., Tan, C.H., Dimler, S., David, J.P. and Ng, J.S. (2014) $1550 \mathrm{~nm} \mathrm{In-}$ GaAs/InAlAs Single Photon Avalanche Diode at Room Temperature. Optics EXpress, 22, 22608-22615. https://doi.org/10.1364/OE.22.022608

[2] Bennett, C.H., Bessette, F., Brassard, G., Salvail, L. and Smolin, J. (1992) Experimental Quantum Cryptography. Journal of Cryptology, 5, 3-28. https://doi.org/10.1007/BF00191318

[3] Kostamovaara, J., Tenhunen, J., Kögler, M., Nissinen, I., Nissinen, J. and Keränen, P. (2013) Fluorescence Suppression in Raman Spectroscopy Using a Time-Gated CMOS SPAD. Optics Express, 21, 31632-31645. https://doi.org/10.1364/OE.21.031632

[4] Warburton, R.E., McCarthy, A., Wallace, A.M., Hernandez-Marin, S., Hadfield, R.H., Nam, S.W. and Buller, G.S. (2007) Subcentimeter Depth Resolution Using a Single-Photon Counting Time-of-Flight Laser Ranging System at $1550 \mathrm{~nm}$ Wavelength. Optics Letters, 32, 2266-2268. https://doi.org/10.1364/OL.32.002266

[5] Soper, S.A., Mattingly, Q.L. and Vegunta, P. (1993) Photon Burst Detection of Single Near-Infrared Fluorescent Molecules. Analytical Chemistry, 65, 740-747. https://doi.org/10.1021/ac00054a015

[6] Rech, I., Luo, G., Ghioni, M., Yang, H., Xie, X.S. and Cova, S. (2004) Photon-Timing Detector Module for Single-Molecule Spectroscopy with 60-ps Resolution. IEEE Journal of Selected Topics in Quantum Electronics, 10, 788-795. https://doi.org/10.1109/JSTQE.2004.833975

[7] Ghioni, M., Cova, S., Zappa, F. and Samori, C. (1996) Compact Active Quenching Circuit for Fast Photon Counting with Avalanche Photodiodes. Review of Scientific Instruments, 67, 3440-3448. https://doi.org/10.1063/1.1147156

[8] Cova, S., Ghioni, M., Lacaita, A., Samori, C. and Zappa, F. (1996) Avalanche Photodiodes and Quenching Circuits for Single-Photon Detection. Applied Optics, 35, 1956-1976. https://doi.org/10.1364/AO.35.001956

[9] Dalla Mora, A., Tosi, A., Tisa, S. and Zappa, F. (2007) Single-Photon Avalanche Diode Model for Circuit Simulations. IEEE Photonics Technology Letters, 19, 1922 1924. https://doi.org/10.1109/LPT.2007.908768 
[10] Mita, R., Palumbo, G. and Fallica, P.G. (2008) Accurate Model for Single-Photon Avalanche Diodes. IET Circuits, Devices \& Systems, 2, 207-212. https://doi.org/10.1049/iet-cds:20070180

[11] Gulinatti, A., Rech, I., Maccagnani, P., Ghioni, M. and Cova, S. (2005) Large-Area Avalanche Diodes for Picosecond Time-Correlated Photon Counting. Solid-State Device Research Conference, ESSDERC, Proceedings of 35 th European, 355-358. https://doi.org/10.1109/ESSDER.2005.1546658

Submit or recommend next manuscript to SCIRP and we will provide best service for you:

Accepting pre-submission inquiries through Email, Facebook, LinkedIn, Twitter, etc. A wide selection of journals (inclusive of 9 subjects, more than 200 journals) Providing 24-hour high-quality service User-friendly online submission system Fair and swift peer-review system Efficient typesetting and proofreading procedure Display of the result of downloads and visits, as well as the number of cited articles Maximum dissemination of your research work

Submit your manuscript at: http://papersubmission.scirp.org/

Or contactopj@scirp.org 